Société d'histoire de la révolution de 1848 et des

révolutions du XIXe siècle

$62 \mid 2021$

Ancêtres

\title{
Alain CORBIN, Terra Incognita. Une histoire de l'ignorance
}

Nicolas Cambon

\section{OpenEdition}

Journals

Édition électronique

URL : https://journals.openedition.org/rh19/7673

DOI : $10.4000 /$ rh 19.7673

ISSN : $1777-5329$

Éditeur

La Société de 1848

Édition imprimée

Date de publication : 20 juin 2021

Pagination : 270-271

ISSN : 1265-1354

Référence électronique

Nicolas Cambon, "Alain CORBIN, Terra Incognita. Une histoire de l'ignorance», Revue d'histoire du XIXe siècle [En ligne], 62 | 2021, mis en ligne le 17 août 2021, consulté le 26 août 2021. URL : http:// journals.openedition.org/rh19/7673 ; DOI : https://doi.org/10.4000/rh19.7673

Ce document a été généré automatiquement le 26 août 2021.

Tous droits réservés 


\title{
Alain CORBIN, Terra Incognita. Une histoire de l'ignorance
}

\author{
Nicolas Cambon
}

\section{RÉFÉRENCE}

Alain CORBIN, Terra Incognita. Une histoire de l'ignorance, Paris, Albin Michel, 2020, 288 p., $21,90 €$.

1 Alain Corbin s'attelle ici à l'histoire d'un objet relativement nouveau ${ }^{1}$, l'ignorance : « Il est impossible de connaître les hommes sans discerner ce qu'ils ne savaient pas » (p. 9) écrit-il dès l'introduction. Mais l'entreprise est vaste. Aussi l'historien circonscrit-il son étude aux savoirs occidentaux sur la Terre, "à l'effacement ou au maintien de ses mystères » (p. 9) du mitan du XVIII ${ }^{\mathrm{e}}$ siècle à la tombée du XIX ${ }^{\mathrm{e}}$ siècle, scrutant l'évolution des connaissances en géographie, géologie, vulcanologie, glaciologie, météorologie et océanologie. Résonnant avec les défis de notre temps, le thème est guetté par la téléologie: le risque est grand de reconduire un récit progressiste, confortant les certitudes d'aujourd'hui. Pourtant, le lecteur se sait en confiance: l'historien du sensible connaît ce risque, contre lequel, dans le sillage de Lucien Febvre, il a depuis longtemps lui-même mis en garde. La grande attention qu'il prête aux expressions affectives que les écrits d'autrefois confient ou trahissent prévient l'anachronisme psychologique, condition de la téléologie.

2 La première partie s'ouvre le $1^{\mathrm{er}}$ novembre 1755 , lors du tremblement de terre qui défigure Lisbonne et exacerbe, chez les contemporains, la conscience de leur ignorance des mécanismes sismiques. Les interprétations se libèrent de la théologie naturelle. Autrefois avertissements du Créateur, les tremblements de terre deviennent des problèmes à résoudre, simples produits des engrenages de l'immense mécanique du «grand horloger». Les affects s'en trouvent modifiés: le sentiment de compassion monte à l'égard des innocentes victimes. De là, l'historien procède au repérage de « la pauvreté des certitudes» et «l'importance des ignorances partagées » durant la 
seconde moitié du XvIII ${ }^{e}$ siècle (p. 31), s'appuyant notamment sur la lecture des entrées de l'Encyclopédie et des dictionnaires du temps. Savants et philosophes s'interrogent aussi bien sur l'âge de la Terre que sur sa structure interne. La planète passe au cours de ce siècle, non sans polémiques, pour plus âgée qu'on ne l'avait cru : si Bossuet situait la Création à moins de 6000 ans, Buffon la renvoie à quelque 110000 années. La question de la nature de la croûte terrestre oppose, quant à elle, la théorie du neptunisme - elle résulterait des sédiments d'un océan originel - à la théorie du plutonisme - elle résulterait de l'activité volcanique. L'indécision domine. Concernant la surface de la Terre, les explorations franco-britanniques dans la «Mer du sud» résorbent les blancs qui mangeaient jusqu'alors les cartes du Vieux Continent. La banquise, infranchissable, interdit l'exploration des pôles et contraint leurs savoirs. Il en va de même pour les abysses dont on ignore tout. L'élément terrestre pour lequel les savoirs progressent le plus vite reste la montagne. Son expérience est d'abord iconographique, avant que l'élite, dans le cadre du Grand Tour, ne commence à visiter les Alpes. Elle est par la suite pourvue de vertus thérapeutiques et "la cure d'air » (p. 75) se développe.

3 Dans un deuxième temps, l'auteur s'arrête sur la période de l'aube du XIX siècle aux années 1850 ; nombre d'ignorances sont confortées. Le chapitre qui étonnera le plus le lecteur est sans doute celui qu'Alain Corbin consacre aux brouillards secs. Entre 1815 et 1818, " partout des "brouillards secs" ont recouvert la terre d'une matière de couleur sombre, au point qu'à midi la lumière du soleil ne la pénétrait pas mieux que celle de la lune ne le fait la nuit» (p. 139). Aux yeux des contemporains, «l'apocalypse semblait se réaliser » (p. 140). William Turner ou Caspar D. Friedrich surent figer cette atmosphère dans leurs toiles. Tout le siècle durant, on ignore son origine, à savoir l'éruption en $1815 \mathrm{du}$ Tambora en Indonésie. La fonte des glaces engendrée permet à Fabian von Bellingshausen d'identifier un nouveau continent en deçà du cercle polaire antarctique. Mais nombreuses sont alors les terrae incognitae concernant les savoirs sur la Terre.

Dans une dernière partie, l'auteur s'emploie à décrire l'accélération du recul du sentiment d'ignorance qui caractérise le second xixe siècle. La pose des câbles au fond de l'Atlantique révèle un terrain accidenté, parsemé çà et là de volcans, mais sans gouffres ni tempêtes comme le supposait Georges Cuvier. S'inaugure l'inventaire de la faune des abysses : en 1869 des créatures jamais vues jusque-là sont remontées à la surface. Dans le domaine des airs, tout un réseau météo-télégraphique se met en place, aiguisant la connaissance de la pression de l'air et des courants aériens. Des journaux relaient auprès des citadins les cartes prévisionnelles qui en découlent. Ce raffinement des connaissances ne concerne pas toutes les ignorances : les pôles demeurent toujours inaccessibles.

Durant toute la période considérée, s'amplifie ce que l'historien nomme le « feuilletage des ignorances» (p.11), les variations de l'état des savoirs d'un individu à un autre. L'auteur est prudent: il reste difficile d'établir ce que savait l'individu moyen. Au xxI siècle, conclut-il, ce constat «s'accroît dans des proportions vertigineuses, et cela pèse sur les relations interpersonnelles» (p. 259). S'immerger dans l'altérité d'une époque, s'en imprégner, mieux comprendre les individus du passé pour mieux distinguer les spécificités des sociétés d'aujourd'hui: tel est en définitive le but de l'ouvrage d'Alain Corbin. Ce livre propose, en somme, un prisme pour comprendre les contemporains de ce long xIX ${ }^{e}$ siècle. 
6 La notion d'ignorance ne va pas sans interroger: se résume-t-elle à une somme d'absences que le futur vient combler? Ne peut-elle pas procéder aussi d'une démarche volontaire, du refus de connaissances jugées anxiogènes? L'auteur signale que l'ignorance a pu être interprétée tel un bienfait. Bernardin de Saint-Pierre l'affirme : «[G]râce à mon ignorance je me laisse aller à l'instinct de mon âme » (p.12). On regrettera que cet aspect n'ait pas été davantage exploré ; l'historien, il est vrai, ne pouvait épuiser son objet en un ouvrage. Il note ainsi que bien des ignorances restent à étudier, telles celles du corps humain. Cette étude serait à coup sûr éclairante, affinant notre compréhension de la perception que les contemporains du passé nourrissaient d'eux-mêmes, dans le sillage de l'entreprise qu'inaugure ici Alain Corbin.

\section{NOTES}

1. Signalons ici l'existence de l'agnotologie (l'étude des ignorances), fondée dans les années 1990 par l'historien des sciences Robert N. Proctor: Robert N. Proctor, Londa Schiebinger (eds.), Agnotology: The Making and Unmaking of Ignorance, Stanford, Stanford University Press, 2008. Plus largement, mentionnons encore les Ignorance studies, apparues outre-Atlantique et connaissant un essor ces dernières années, notamment: Matthias Gross, Linsey McGoey (eds.), Routledge International Handbook of Ignorance Studies, Londres, Routledge, 2015; Adriana Mica, Anna Horolets, Mikołaj Pawlak, Paweł Kubicki, Ignorance and Change: Anticipatory Knowledge and the European Refugee Crisis, Londres, Routledge, 2020. 